\title{
NORMAL AND HERMITIAN COMPOSITION OPERATORS
}

\section{RAJ KISHOR SINGH}

ABSTRACT. Let $C_{\phi}$ be a composition operator on $L^{2}(\lambda)$. Some conditions under which $C_{\phi}$ is an isometry and Hermitian are investigated in this paper. Some study of normal composition operators is also made.

1. Introduction. This paper is a continuation of [3]. Let $\lambda$ be a $\sigma$-finite measure on a set $X$, and let $\phi$ be a measurable transformation on $X$ into itself. Then define a composition transformation $C_{\phi}$ on $L^{2}(\lambda)$ as $C_{\phi} f=$ $f \circ \phi$ for every $f$ in $L^{2}(\lambda)$. If $C_{\phi}$ is bounded, then it is called a composition operator. In [3] we have characterized all quasinormal composition operators. Our purpose in this paper is to report on normal and Hermitian composition operators. In $\$ 2$ we shall investigate all isometric composition operators, and we shall also prove that in certain cases quasinormality implies normality. $\$ 3$ is devoted to the characterization of Hermitian composition operators. This class of operators has been characterized by Ridge [2]. Since our proofs and approaches are entirely different from Ridge's, they are given here.

2. Isometric and normal composition operators. If $\phi: X \rightarrow X$ is a measurable transformation, then $\lambda \phi^{-1}$ is a measure on $X$. Also if $C_{\phi}$ is a composition operator, then $\lambda \phi^{-1}$ is absolutely continuous with respect to $\lambda$. Let us denote the Radon-Nikodym derivative of $\lambda \phi^{-1}$ by $f_{0}$. Ridge [2] proves that if $f_{0}=1$ (a.e.), then $C_{\phi}$ is an isometry. This condition is also necessary, as the following theorem shows.

Theorem 1. Let $C_{\phi}$ be a composition operator on $L^{2}(\lambda)$. Then a necessary and sufficient condition that $C_{\phi}$ be an isometry is that $f_{0}=1$ (a.e.).

Proof. The proof is a trivial consequence of the relation $C_{\phi}^{*} C_{\phi}=M_{f_{0}}$, where $M_{f_{0}}$ is the multiplication operator induced by $f_{0}$.

Received by the editors November 29, 1973.

AMS (MOS) subject classifications (1970). Primary 47B15; Secondary 47B15.

Key words and phrases. Composition operators, isometry, normal and Hermitian operators, quasinormal operators. 
It is well known that every normal operator is quasinormal, but the converse is not true. However, in the case of composition operators induced by one-to-one (a.e.) measurable transformations, the converse is also true. This is shown in the following theorem.

Theorem 2. Let $C_{\phi}$ be a composition operator on $L^{2}(\lambda)$ induced by a one-to-one (a.e.) transformation $\phi$. Then $C_{\phi}$ is quasinormal if and only if it is normal.

Proof. First we claim that if $\phi$ is one-to-one (a.e.), then the range of $C_{\phi}$ is dense. We shall outline the proof as follows. Since $\phi$ is a one-toone (a.e.), there exists a measurable transformation $\psi$ such that $(\psi \circ \phi)(x)$ $=x$ (a.e.). If $\lambda(X)<\infty$, then the range of $C_{\phi}$ is obviously dense as it contains all characteristic functions, and hence all simple functions. So let $\lambda(X)=\infty$. Since $\lambda$ is $\sigma$-finite, we can write $X=\bigcup_{i=1}^{\infty} X_{i}$, where $X_{i}$ 's are disjoint and $\lambda\left(X_{i}\right)<\infty$ for every $i$. Let $X_{E}$ (the characteristic function of the set $E$ ) be in $L^{2}(\lambda)$. Then

$$
X_{\psi^{-1}(E)}=\sum_{i=1}^{\infty} X_{F_{i}},
$$

where $F_{i}=\psi^{-1}(E) \cap X_{i}$. Consider the sum $\sum_{i=1}^{\infty} X_{F_{i}} \circ \phi$. If $x \in X$, it is clear that $\phi(x) \in F_{i}$ for some $i$ iff $\phi(x) \in \psi^{-1}(E)$. Hence the above sum converges to $X_{E}$ (a.e.). By the Lebesgue dominated convergence theorem, it converges to $X_{E}$ in $L^{2}$-norm, and hence $X_{E}$ is in the closure of the range of $C_{\phi}$. This is enough to show that the range of $C_{\phi}$ is dense in $L^{2}(\lambda)$.

Now we shall prove the theorem. Suppose $C_{\phi}$ is normal; then it follows trivially that it is quasinormal. On the other hand, suppose $C_{\phi}$ is quasinormal. We know that $C_{\phi}^{*} C_{\phi}=M_{f_{0}}$. Hence

$$
C_{\phi} C_{\phi}^{*} C_{\phi}=C_{\phi} M_{f_{0}}
$$

and since $C_{\phi}$ is quasinormal, we get $C_{\phi} C_{\phi}^{*} C_{\phi}=M_{f_{0}} C_{\phi}$. This shows that $C_{\phi} C_{\phi}^{*}$ and $M_{f_{0}}$ agree on the range of $C_{\phi}$, which is dense in $L^{2}(\lambda)$. Hence

$$
C_{\phi} C_{\phi}^{*}=M_{f_{0}}=C_{\phi}^{*} C_{\phi} \text {. }
$$

This proves that $C_{\phi}$ is normal.

3. Hermitian composition operators. Hermitian composition operators are characterized by Ridge [2], where he uses the techniques depending on 
the spectrum of the operator. In Theorem 3 a different characterisation, using a simple and different method, is given.

Theorem 3. Let $C_{\phi}$ be a composition operator on $L^{2}(\lambda)$. Then $C_{\phi}$ is Hermitian if and only if $f_{0}=1$ (a.e.) and $(\phi \circ \phi)(x)=x$ (a.e.).

Proof. Suppose $C_{\phi}$ is Hermitian. Then $C_{\phi}=C_{\phi}^{*}$. Since $C_{\phi}^{*} C_{\phi}=M_{f_{0}}$, we get $C_{\phi \circ \phi}=C_{\phi} C_{\phi}=M_{f_{0}}$. We can write $X=\bigcup_{n=1}^{\infty} E_{n}$, where $\lambda\left(E_{n}\right)<0_{\infty}$ for each $n$, and $E_{n} \subset E_{m}$ if $m>n$. If $f_{n}=X_{E_{n}}$, then we obtain $C_{\phi \circ \phi} f_{n}=$ $M_{f_{0}} f_{n}$. Equivalently, $X_{(\phi \circ \phi)^{-1}\left(E_{n}\right)}=f_{0} \cdot X_{E_{n}}$. Since $\bigcup_{n=1}^{\infty}(\phi \circ \phi)^{-1}\left(E_{n}\right)$ $=X$, we can conclude that $f_{0}=1$ (a.e.) and also $(\phi \circ \phi)(x)=x$ (a.e.).

Conversely, suppose $f_{0}=1$ (a.e.) and $(\phi \circ \phi)(x)=x$ (a.e.). Then

$$
C_{\phi}^{*} C_{\phi}=.1_{f_{0}}=I \quad \text { (the identity operator). }
$$

Multiplying both sides on the right by $C_{\phi}$ we get

$$
C_{\phi}^{*} C_{\phi} C_{\phi}=C_{\phi}, \quad C_{\phi}^{*} C_{\phi \circ \phi}=C_{\phi}, \quad C_{\phi}^{*}=C_{\phi} .
$$

This shows that $C_{\phi}$ is Hermitian.

Corollary. If $C_{\phi}$ is a composition operator on $L^{2}(\lambda)$, then $C_{\phi}$ is a projection if and only if $C_{\phi}=I$.

Corollary. $C_{\phi}$ is Hermitian implies $C_{\phi}$ is an isometry.

\section{REFERENCES}

1. P. R. Halmos, A Hilbert space problem book, Van Nostrand, Princeton, N. J., 1967. MR $34 \# 8178$.

2. W. C. Ridge, Composition operators, Thesis, Indiana University, Bloomington, Ind., 1969.

3. R. K. Singh, Compact and quasinormal composition operators, Proc. Amer. Math. Soc. 45 (1974), 80-82.

DEPARTMENT OF MATHEMATICS, UNIVERSITY OF JAMMU, JAMMU, INDIA 